\title{
A Sparse Kernel Representation Method for Image Classification
}

\author{
Shuyuan Yang \\ Department of electronic engineering \\ Key Lab of Intelligent Perception and Image Understanding \\ of Ministry of Education \\ Xi'an, China \\ syyang@xidian.edu.cn
}

\author{
Yue Han, XiangRong Zhang \\ Department of electronic engineering \\ Key Lab of Intelligent Perception and Image Understanding \\ of Ministry of Education \\ $\mathrm{Xi}$ 'an, China \\ 576087010@qq.com
}

\begin{abstract}
In this paper, we propose a sparse kernel representation classification algorithm (SKRC) for images classification and recognition. The training dictionary is composed by labeled samples directly, and both training dictionary and testing sample are mapped into feature space from original sample space by the sparse kernel which employs the "center" samples matrix constructed by a method similar to $k$-means clustering. Then in the feature space, the basic sparse representation based classification method is employed. We test our proposed algorithm on some different public database, and the results show that our proposed method can achieve higher classification accuracy without much time consumed.
\end{abstract}

Keywords-sparse kernel; sparse representation; classification

\section{INTRODUCTION}

Image classification technology has grown fast over the past decade, and among them there are three kinds of methods: unsupervised methods [1-3], semi-supervised methods[4-6] and supervised methods[7-9]. All of these methods have their own positives and negatives. The unsupervised methods such as k-means algorithm, don't need to have any labeled data, but heavily rely on the initialization, and always achieve unsatisfactory result. The semi-supervised classification methods always utilize both labeled data and the information conveyed by the marginal distribution of the unlabeled samples to boost the algorithmic performance when the lack of enough labeled training data in real-world applications, for example: graph based semi-supervised learning and generative models. Each methods have their own disadvantages: graph based semi-supervised learning is sensitive to the graph construction and generative models must know the mixture distribution model of training data, which can't be satisfied in the real applications. The supervised methods such as SVM, can achieve higher recognition accuracy, however, they require long training process and the model parameter is difficult to choose.

Sparse coding can be traced back to the research by David Hubel and Toresten Wiesel in 1959[10]. Nowadays many algorithms about sparsity and sparse coding have been proposed. With the generation of the theory of compressed sensing [11], sparse coding has become the hotspot once again. All kinds of sparse coding algorithm have been widely used in image denoising[12][13], blind source separation[14][15], speech signal processing[16][17], image feature extraction[18][19] and pattern recognition[20][21], etc.

As a kind of methods of sparse coding, J.Wright proposed the sparse representation classification algorithm (SRC) for human face recognition [20], which was robust to occlusion. Because some data samples are linearly inseparable in the sample space, Shenghua Gao proposed the kernel sparse representation classification algorithm (KSRC) [21], in which algorithm data samples were mapped into the feature space and the algorithm of SRC were carried out in the feature space, but calculating kernel function always costs too much time. In order to reduce recognition time without classification accuracy loss, we propose a new sparse kernel representation algorithm for image classification. By choosing certain number of training samples to construct the "center" samples matrix, we map both the training samples and the testing samples into feature space by the kernel function, followed by the basic sparse representation classification algorithm (SRC). Experimental results demonstrate that when compared with other algorithms, our method is characteristics of 1) satisfied classification accuracy with its counterparts; 2) rapid classification process.

The remainder of this is organized as follows: In Section 2, we give an overview of the SRC algorithm and the KSRC algorithm. Section 3 presents our proposed method in detail, followed by some experiments results in Section 4. Our conclusion of this paper is shown in Section 5.

\section{SPARSE REPRESENTATION CLASSIFICATION}

\section{A. Sparse representation classification}

SRC employs the principle that any test sample can be represented as a linear representation of the training samples from the same class approximately, therefore it can be considered as a sparse linear combination of training samples from all classes [7]. 
Suppose the training samples are in the finite dimensional, real inner - product space $\mathbf{R}^{N}$, and all the training samples are used to serve as a dictionary $\mathbf{D}=\left[\mathbf{d}_{1,1}, \mathbf{d}_{1,2}, \ldots \mathbf{d}_{1, n_{1}}, . . \mathbf{d}_{i, 1}, . . \mathbf{d}_{i, j}, \ldots \mathbf{d}_{i, n_{i}}, \ldots \mathbf{d}_{l, 1}, \mathbf{d}_{l, 2}, \ldots, \mathbf{d}_{l, n_{l}}\right] \in \mathbf{R}^{N \times G}$ where $\mathbf{d}_{i, j}$ represents the $j^{\text {th }}$ training sample belongs to the $i^{\text {th }}$ class,$n_{i}$ means the number of training samples in the $i^{\text {th }}$ class,$l$ is the total number of classes and $G=\sum_{i=1}^{l} n_{i} \cdot \mathbf{y}$ is the test sample whose label is to be determined. For any test sample belongs to some class in the dictionary, we can represent it as the following formula:

$$
\mathbf{y}=\mathbf{D} \boldsymbol{\alpha}
$$

where $\quad \boldsymbol{\alpha}=\left[0,0, \ldots, 0, \alpha_{i, 1}, \alpha_{i, 2}, . . \alpha_{i, n_{i}}, 0, \ldots, 0\right]^{T} \quad$ is $\quad$ a sparse coefficient vector whose entries are zero except those associated with the $i^{\text {th }}$ class. We can obtain $\hat{\boldsymbol{\alpha}}$ by solving such a $l_{0}$-norm minimization problem shown in (2),

$$
\hat{\boldsymbol{\alpha}}=\arg \min _{\boldsymbol{\alpha}}\|\boldsymbol{\alpha}\|_{0} \quad \text { s.t. } \mathbf{y}=\mathbf{D} \boldsymbol{\alpha}
$$

Recent development in the theory of compressed sensing [11] reveals that if the coefficient $\hat{\boldsymbol{\alpha}}$ is sparse enough, the solution in (2) can be replaced by an $l_{1}$-norm substitution as following:

$$
\hat{\boldsymbol{\alpha}}=\arg \min _{\boldsymbol{\alpha}}\|\boldsymbol{\alpha}\|_{1} \quad \text { s.t. } \mathbf{y}=\mathbf{D} \boldsymbol{\alpha}
$$

This problem can be solved in polynomial time by standard linear programming methods [22].

After estimating the coefficients vector $\hat{\boldsymbol{\alpha}}$, we define the function $\boldsymbol{\delta}_{i}(\hat{\boldsymbol{\alpha}})$ as a new vector whose nonzero entries are the entries in $\hat{\boldsymbol{\alpha}}$ that are associated with the $i^{\text {th }}$ class. After that, we can classify $\mathbf{y}$ to a class that has the minimal residual, that is,

$$
\operatorname{identity}(\mathbf{y})=\arg \min \left\|\mathbf{y}-\mathbf{D} \boldsymbol{\delta}_{i}(\hat{\boldsymbol{\alpha}})\right\|_{2}
$$

The SRC algorithm has been successfully applied to face recognition [7] and targets classification of SAR images [23].

\section{B. Kernel sparse representation classification}

The dictionary in SRC is established using the training samples without some complicated training processing. However, this method can not achieve a satisfactory classification ratio when data samples are nonlinear in the original sample space especially.

The key point of kernel methods is that they exploit an efficient way for a nonlinear mapping from the finite dimensional sample space to a very high dimensional Hilbert space called feature space. Let $\boldsymbol{\Phi}: \mathbf{R}^{N} \rightarrow \mathbf{R}^{F}$ be a non-linear mapping from the data space to feature space. By this mapping, a vector $\mathbf{m} \in \mathbf{R}^{N}$ becomes $\Phi(\mathbf{m}) \in \mathbf{R}^{F}$. In most cases, we don't know the specific form of the mapping function $\boldsymbol{\Phi}$. However according to the kernel trick in [24], the innerproduct of two mapped samples in the feature space can be represented by the kernel function of these two samples in the original sample space, which can be represented as following

$$
k(\mathbf{m}, \mathbf{n})=\boldsymbol{\Phi}(\mathbf{m})^{T} \boldsymbol{\Phi}(\mathbf{n})=(\boldsymbol{\Phi}(\mathbf{m}) \cdot \boldsymbol{\Phi}(\mathbf{n}))
$$

where $(\cdot)$ means the dot-products of vector $\mathbf{m}$ and vector $\mathbf{n}$.

There are some kernel functions which are commonly used:

Linear kernel:

$$
k(\mathbf{m}, \mathbf{n})=(\mathbf{m} \cdot \mathbf{n})
$$

Polynomial kernel:

$$
k(\mathbf{m}, \mathbf{n})=((\mathbf{m} \cdot \mathbf{n})+c)^{d}, d \in 1,2, \ldots
$$

Sigmoidal kernel:

$$
k(\mathbf{m}, \mathbf{n})=\tanh (a(\mathbf{m} \cdot \mathbf{n})+b)
$$

Gaussian RBF kernel:

$$
k(\mathbf{m}, \mathbf{n})=\exp \left(-\|\mathbf{m}-\mathbf{n}\|^{2} / 2 p^{2}\right)
$$

The kernel sparse representation method (KSRC) is based on the mapping of both training samples and testing samples into feature space by the kernel trick and solve an $l_{1}$-norm minimization problem in the feature space with new samples.

Mapping training samples and testing samples into feature space we can get

$$
\Phi(\mathbf{y})=\Phi(\mathbf{D}) \boldsymbol{\alpha}_{1}
$$

where $\boldsymbol{\alpha}_{1}$ is the new sparse coefficient in the feature space. Multiplying $\Phi(\mathbf{D})^{T}$ to both sides of equation (10), we can obtain the following:

$$
\Phi(\mathbf{D})^{T} \Phi(\mathbf{y})=\Phi(\mathbf{D})^{T} \Phi(\mathbf{D}) \boldsymbol{\alpha}_{1}
$$

According to the definition of inner-product, we can get

$$
(\Phi(\mathbf{D}) \cdot \Phi(\mathbf{y}))=(\Phi(\mathbf{D}) \cdot \Phi(\mathbf{D})) \boldsymbol{\alpha}_{1}
$$

Using the kernel trick, equation (12) can be rewritten as 


$$
\mathbf{K}(\mathbf{D}, \mathbf{y})=\mathbf{K}(\mathbf{D}, \mathbf{D}) \boldsymbol{\alpha}_{1}
$$

where $\quad \mathbf{K}(\mathbf{D}, \mathbf{y})=\left[k\left(\mathbf{d}_{1,1}, \mathbf{y}\right) k\left(\mathbf{d}_{1,2}, \mathbf{y}\right) \ldots . . k\left(\mathbf{d}_{l, n_{l}}, \mathbf{y}\right)\right]^{T} \in \mathbf{R}^{G \times 1}$ represents the kernel matrix which is composed by the value of kernel function between every column $\mathbf{d}_{i, j}$ in the dictionary $\mathbf{D}$ and the testing sample $\mathbf{y}$, the kernel matrix $\mathbf{K}(\mathbf{D}, \mathbf{D})$ can be obtained by the value of kernel function between any column in $\mathbf{D}$, which can be written as,

$\mathbf{K}(\mathbf{D}, \mathbf{D})=\left[\begin{array}{cccc}k\left(\mathbf{d}_{1,1}, d_{1,1}\right) & k\left(\mathbf{d}_{1,1}, \mathbf{d}_{1,2}\right) & \ldots & k\left(\mathbf{d}_{1,1}, \mathbf{d}_{l, n_{l}}\right) \\ k\left(\mathbf{d}_{1,2}, d_{1,1}\right) & k\left(\mathbf{d}_{1,2}, \mathbf{d}_{1,2}\right) & \ldots & k\left(\mathbf{d}_{1,2}, \mathbf{d}_{l, n_{l}}\right) \\ \ldots & \ldots & \ldots & . . \\ k\left(\mathbf{d}_{l, n_{l}}, d_{1,1}\right) & k\left(\mathbf{d}_{l, n_{l}}, \mathbf{d}_{1,2}\right) & \ldots & k\left(\mathbf{d}_{l, n_{l}}, \mathbf{d}_{l, n_{l}}\right)\end{array}\right] \in R^{G \times G}$

where $k\left(\mathbf{d}_{i, j}, \mathbf{d}_{p, q}\right)$ means the value of kernel function between sample $\mathbf{d}_{i, j}$ and sample $\mathbf{d}_{p, q}, i, p=1, \ldots, l \quad j, q=1, \ldots, n_{l}$.

However, in some practical applications, due to $G$, the dimension of new samples in (13) is very large, we need to decrease the dimensionality of the mapped samples. By using some dimensionality reduction approaches, such as random sampling used in the compressive sensing and so on, the formula (13) can be reformulated as:

$$
\tilde{\mathbf{K}}(\mathbf{D}, \mathbf{y})=\tilde{\mathbf{K}}(\mathbf{D}, \mathbf{D}) \boldsymbol{\alpha}_{2}
$$

where $\boldsymbol{\alpha}_{2}$ is the sparse coefficient vector under the framework of KSRC. The formula (15) can be substitute by a $l_{1}$-norm minimization problem shown in (16).

$$
\hat{\boldsymbol{\alpha}}_{2}=\arg \min _{\boldsymbol{\alpha}_{2}}\left\|\boldsymbol{\alpha}_{2}\right\|_{1} \quad \text { s.t. } \tilde{\mathbf{K}}(\mathbf{D}, \mathbf{y})=\tilde{\mathbf{K}}(\mathbf{D}, \mathbf{D}) \boldsymbol{\alpha}_{2}
$$

\section{SPARSE KERNEL REPRESENTATION CLASSIFICATION}

Although the classification accuracy of KSRC is obviously higher than that of SRC, KSRC is not always applicable to practical applications because we must calculate $G$ times of kernel function for each training sample and testing sample, which is very time consuming.

In order to improve the classification accuracy of KSRC and overcome the disadvantage of time consuming, we propose a new sparse kernel representation method. In our proposed SKRC, we only calculate $u(u<<G)$ kernel function using a "center" samples matrix $\mathbf{C}$, which is constructed from the training samples by an idea similar to kmeans clustering [25]. The specific value of $u$ depend on the dimensionality decreased. We just choose the value of $u$ which is equal to or higher than the dimensionality required. The detailed procedure for the "center" samples matrix construction is listed in Algorithm 1. The number of "center"samples to choose in each class is determined by the proportion of training samples in each class as long as $\sum_{i=1}^{l} \mathbf{x}_{i}=u$.

Algorithm 1 : the "center" samples matrix construction

Inputs: the dictionary D , the number of "center" samples $u$, the total number of classes $l$

Outputs: the "center"samples matrix $\mathbf{C}$

1 For the $i^{\text {th }}$ class training samples in the dictionary $\mathbf{D}$, $i=1,2, \ldots l$

a) Compute the mean vector $\mathbf{0}_{i}$;

b) According to ascending order of the distance, choose first $x_{i}$ training samples $\left[\mathbf{d}_{i, 1}^{\prime} \mathbf{d}_{i, 2}^{\prime} \ldots \mathbf{d}_{i, x_{i}}^{\prime}\right]$;

c) Obtain the "center" samples $\mathbf{c}_{i}=\left[\begin{array}{l}\mathbf{o}_{i} \\ \mathbf{d}_{i, 1}^{\prime}\end{array} \mathbf{d}_{i, 2}^{\prime} \ldots . . . \mathbf{d}_{i, x_{i}}^{\prime}\right]$.

2 Obtain the "center" samples matrix $\mathbf{C}=\left[\mathbf{c}_{1}, \mathbf{c}_{2}, \ldots \mathbf{c}_{l}\right]$

Using the "center" samples matrix to map the dictionary and testing sample, we can get the following equation:

$$
\mathbf{K}(\mathbf{C}, \mathbf{y})=\mathbf{K}(\mathbf{C}, \mathbf{D}) \boldsymbol{\alpha}_{1}
$$

Where $\mathbf{K}(\mathbf{C}, \mathbf{y}) \in \mathrm{R}^{u \times 1}$ represents the kernel function between every "center" sample in $\mathbf{C}$ and the testing sample $\mathbf{y}$, and it can be considered as the new testing sample in the feature space.

$$
\begin{aligned}
\mathbf{K}(\mathbf{C}, \mathbf{y})=\left[k\left(\mathbf{o}_{1}, \mathbf{y}\right) k\left(\mathbf{d}_{1,1}^{\prime}, \mathbf{y}\right) \ldots k\left(\mathbf{d}_{1, x_{1}}^{\prime}, \mathbf{y}\right) \ldots\right. \\
\left.\ldots k\left(\mathbf{o}_{l}, \mathbf{y}\right) k\left(\mathbf{d}_{l, 1}^{\prime}, \mathbf{y}\right) \ldots k\left(\mathbf{d}_{l, x_{l}}^{\prime}, \mathbf{y}\right)\right]^{T}
\end{aligned}
$$

Similarly, we can get the following:

$$
\mathbf{K}(\mathbf{C}, \mathbf{D})=\left[\begin{array}{cccc}
k\left(\mathbf{o}_{1}, \mathbf{d}_{1,1}\right) & k\left(\mathbf{o}_{1}, \mathbf{d}_{1,2}\right) & \ldots & k\left(\mathbf{o}_{1}, \mathbf{d}_{l, n_{n}}\right) \\
k\left(\mathbf{d}_{1,1}^{\prime}, \mathbf{d}_{1,1}\right) & k\left(\mathbf{d}_{1,1}^{\prime}, \mathbf{d}_{1,2}\right) & \ldots & k\left(\mathbf{d}_{1,1}^{\prime}, \mathbf{d}_{l, n_{l}}\right) \\
\ldots & \ldots & \ldots & . . \\
k\left(\mathbf{d}_{l, x_{l}}^{\prime}, \mathbf{d}_{1,1}\right) & k\left(\mathbf{d}_{l, x_{i}}^{\prime}, \mathbf{d}_{1,2}\right) & \ldots & k\left(\mathbf{d}_{l, x_{i}}^{\prime}, \mathbf{d}_{l, n_{l}}\right)
\end{array}\right] \in \mathbf{R}^{\imath \prec G}
$$

where $\mathbf{K}(\mathbf{C}, \mathbf{D})$ can be considered as the new dictionary in the feature space.

Choosing the "center" samples matrix instead of all training samples to construct sparse kernel function has the following two advantages. Firstly, because the dimensionality decreased is rather small so as to the number of samples in the "center" samples matrix $C$ is much fewer than the number in the dictionary, we can save lots of time to compute the kernel function. Secondly, elements in matrix $\mathbf{K}$ can represent the similarity among samples. The value is larger means that there 
has stronger affinity between two samples. The kernel matrix obtained by the sparse kernel function will enhance the similarity of samples in the same class and the diversity of samples in different class, which is in favour of follow-up classification.

If the dimensionality is still larger than the required, we can reduce the dimension of new training samples and testing samples in feature space by some dimensionality reduction technologies, as follows,

$$
\tilde{\mathbf{K}}(\mathbf{C}, \mathbf{y})=\tilde{\mathbf{K}}(\mathbf{C}, \mathbf{D}) \boldsymbol{\alpha}_{2}
$$

where $\mathbf{K}(\mathbf{C}, \mathbf{y})$ can be considered as the decreased testing sample and $\mathbf{K}(\mathbf{C}, \mathbf{D})$ is the decreased dictionary.

After that, we can solve the $l_{1}$ minimization to estimate the label of testing sample. The detailed processing of the SKRC algorithm is listed in Algorithm 2.

\footnotetext{
Algorithm 2: sparse kernel representation classification

Inputs: all training samples, the total number of classes $l$ the testing sample $\mathbf{y}$
}

Outputs: the label of testing sample

1 Construct the dictionary $\mathbf{D}$ by training samples arranged by classes.

2 Normalize samples in $\mathbf{D}$ and the testing sample $\mathbf{y}$ to have unit $l_{1}$-norm.

3 Map $\mathbf{D}$ and $\mathbf{y}$ by the sparse kernel function constructed by the algorithm in Algorithm1 to get the new dictionary $\mathbf{K}(C, \mathbf{D})$ and new testing sample $\mathbf{K}(C, \mathbf{y})$.

4 Normalize samples in $\mathbf{K}(C, \mathbf{D})$ to have unit $l_{1}$-norm.

5 Decrease the dimensionality of samples in $\mathbf{K}(C, \mathbf{D})$ and the new testing samples $\mathbf{K}(C, \mathbf{y})$ to get the decreased samples

$$
\tilde{\mathbf{K}}(C, \mathbf{D}) \text { and } \tilde{\mathbf{K}}(C, \mathbf{y}) .
$$

6 solve the $l_{1}$ minimization problem

$$
\hat{\boldsymbol{\alpha}}_{2}=\arg \min _{\boldsymbol{\alpha}_{2}}\left\|\boldsymbol{\alpha}_{2}\right\|_{1} \text { s.t. } \tilde{\mathbf{K}}(C, \mathbf{y})=\tilde{\mathbf{K}}(C, \mathbf{D}) \boldsymbol{\alpha}_{2}
$$

7 Estimate the label of testing sample by

$$
\operatorname{identity}(\mathbf{y})=\arg \min \left\|\tilde{\mathbf{K}}(C, \mathbf{y})-\tilde{\mathbf{K}}(C, \mathbf{D}) \boldsymbol{\delta}_{i}\left(\hat{\boldsymbol{\alpha}}_{2}\right)\right\|_{2}
$$

\section{EXPERIMENTAL RESULTS}

In this section, some experiments are taken to investigate the performance of our proposed method. Firstly, we give a brief introduction about databases we used. Secondly, some experiments on different databases are given to show the superiority of our algorithm. Finally, we take the ORL database [26] for example to analyze some details in the SKRC algorithm. Basis Pursuit (BP) [27] is used to solve the problem of $l_{1}$ minimization. In our proposed method and the KSRC algorithm, the parameter of kernel function is determined by experiments at the lowest dimensionality, and the parameter at other dimensionality is selected near the one at the lowest. All experiments are taken by 20 times independently and the averaged results are calculated.

\section{A. Databases introduction}

We gathered two popular face databases including ORL and extend Yale-B database [28], and two handwritten digit databases including USPS[29] and MNIST[30].

The ORL database contains 400 images of 40 persons, and each person has 10 images which are taken by different facial expression or configuration and have the size of $92 \times 112$ pixels. The extend Yale-B database contains 2414 images of 38 persons, where each person has about 64 images taken by different facial expression or configuration. The USPS database consists of $16^{*} 16$ handwritten digits. 200 samples are randomly selected from each category of handwriting samples (0-9) in the USPS handwriting training database for our test. MNIST database consists of 70000 handwritten digits from 0 to 9 , and each image is cropped and normalized to the size of $28 \times 28$.We also select 200 samples from each category randomly. Some images in four databases are shown in Figure.1.

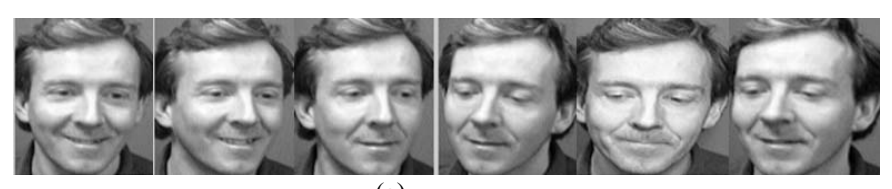

(a)

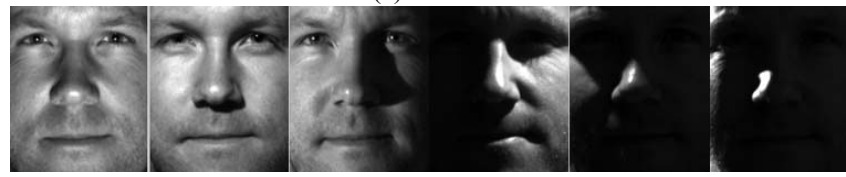

(b)
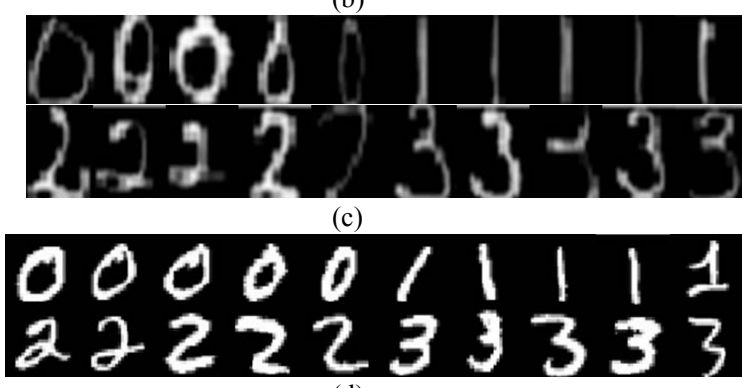

(d)

Figure 1. Images in four databases.(a) ORL, (b) extend Yale-B,(c) USPS,(d) MNIST.

\section{B. Experimental results}

In this section, for all these 5 databases, we select half data from each class served as training samples and the left are served as testing samples. We compare the proposed method with SRC and KSRC under different dimensionality which is reduced by random sampling with Gaussian random measurement matrix. 
In two face databases, dimensionality of samples is reduced to $20,30,40,50,60,70$ and 80 , and samples in handwritten digit databases is reduced to $10,15,20,25,30,35$ and 40 . The number of "center" samples at different dimensionality and classification result can be seen in table I respectively.

TABLE I. EXPERIMENT RESULTS IN DIFFERENT DATABASES(A) ORL, (B) EXTEND YALE-B,(C) USPS,(D) MNIST.

\begin{tabular}{|c|c|c|c|c|c|c|c|}
\hline \multirow{2}{*}{ Dim } & \multirow{2}{*}{$\begin{array}{c}\text { Number } \\
\text { of } \\
\text { "center" } \\
\text { samples }\end{array}$} & \multicolumn{2}{|c|}{ Our method } & \multicolumn{2}{|c|}{ KSRC } & \multicolumn{2}{|c|}{$\mathrm{SRC}$} \\
\hline & & accuracy & time & accuracy & time & accuracy & time \\
\hline 20 & 40 & 91.60 & 9.71 & 88.67 & 11.42 & 57.50 & 6.53 \\
\hline 30 & 40 & 93.38 & 11.32 & 90.98 & 13.04 & 66.48 & 7.83 \\
\hline 40 & 40 & 93.82 & 10.67 & 92.50 & 14.20 & 71.25 & 8.10 \\
\hline 50 & 80 & 93.00 & 13.42 & 92.68 & 14.56 & 73.10 & 9.88 \\
\hline 60 & 80 & 94.20 & 13.35 & 92.97 & 15,34 & 74.20 & 11.12 \\
\hline 70 & 80 & 94.00 & 18.23 & 93.55 & 17.22 & 73.83 & 13.44 \\
\hline 80 & 80 & 94.37 & 17.60 & 93.05 & 19.99 & 74.60 & 15.29 \\
\hline \multicolumn{8}{|c|}{ (A) } \\
\hline \multirow{2}{*}{ Dim } & $\begin{array}{c}\text { Number } \\
\text { of }\end{array}$ & \multicolumn{2}{|c|}{ Our method } & \multicolumn{2}{|c|}{ KSRC } & \multicolumn{2}{|c|}{ SRC } \\
\hline & $\begin{array}{l}\text { center } \\
\text { samples }\end{array}$ & accuracy & time & accuracy & time & accuracy & time \\
\hline 20 & 38 & 85.99 & 214.64 & 78.16 & 384.36 & 70,56 & 137.47 \\
\hline 30 & 38 & 91.38 & 230.96 & 86.99 & 392.35 & 83.28 & 187.60 \\
\hline 40 & 76 & 92.78 & 242.73 & 90.97 & 445.84 & 88.51 & 227.75 \\
\hline 50 & 76 & 94.24 & 453.62 & 93.15 & 503.93 & 90.71 & 311.06 \\
\hline 60 & 76 & 94.55 & 455.66 & 94.08 & 586.21 & 91.64 & 337.37 \\
\hline 70 & 76 & 95.48 & 523.70 & 94.72 & 746.97 & 93.14 & 403.32 \\
\hline 80 & 114 & 95.88 & 616.69 & 95.33 & 796.69 & 93.34 & 433.23 \\
\hline
\end{tabular}

\begin{tabular}{cccccccc}
\hline & \multicolumn{9}{c}{ (B) } \\
\hline & $\begin{array}{c}\text { Number } \\
\text { of } \\
\text { Dim }\end{array}$ & \multicolumn{2}{c}{ Our method } & \multicolumn{2}{c}{ KSRC } & \multicolumn{2}{c}{ SRC } \\
\cline { 3 - 9 } & $\begin{array}{c}\text { center" } \\
\text { samples }\end{array}$ & accuracy & time & accuracy & time & accuracy & time \\
\hline 10 & 10 & $\mathbf{8 7 . 1 9}$ & 58.93 & 81.83 & 113.33 & 71.22 & $\mathbf{4 7 . 5 2}$ \\
15 & 20 & $\mathbf{9 0 . 9 8}$ & 77.54 & 89.15 & 113.60 & 82.76 & $\mathbf{5 2 . 7 0}$ \\
20 & 20 & $\mathbf{9 2 . 2 7}$ & 74.53 & 91.81 & 121.58 & 88.00 & $\mathbf{5 9 . 1 7}$ \\
25 & 30 & $\mathbf{9 2 . 9 1}$ & 94.49 & 92.54 & 131.02 & 90.50 & $\mathbf{6 3 . 2 5}$ \\
30 & 30 & $\mathbf{9 3 . 5 2}$ & 86.08 & 93.24 & 142.77 & 91.79 & $\mathbf{7 0 . 0 9}$ \\
35 & 40 & $\mathbf{9 3 . 8 3}$ & 114.65 & 93.32 & 156.27 & 92.33 & $\mathbf{8 1 . 4 7}$ \\
40 & 40 & $\mathbf{9 4 . 0 9}$ & 104.46 & 93.90 & 177.07 & 92.44 & $\mathbf{8 7 . 1 9}$ \\
\hline
\end{tabular}

\begin{tabular}{|c|c|c|c|c|c|c|c|}
\hline \multirow{2}{*}{ Dim } & \multirow{2}{*}{$\begin{array}{c}\text { Number } \\
\text { of } \\
\text { "center" } \\
\text { samples }\end{array}$} & \multicolumn{2}{|c|}{ Our method } & \multicolumn{2}{|c|}{ KSRC } & \multicolumn{2}{|c|}{ SRC } \\
\hline & & accuracy & time & accuracy & time & accuracy & time \\
\hline 10 & 10 & 85.32 & 45.09 & 74.92 & 135.91 & 50.03 & 39.65 \\
\hline 15 & 20 & 85.66 & 58.80 & 81.60 & 135.92 & 65.05 & 43.25 \\
\hline 20 & 20 & 88.66 & 54.07 & 85.97 & 134.84 & 72.24 & 44.76 \\
\hline 25 & 30 & 88.68 & 69.74 & 88.03 & 150.00 & 77.17 & 48.93 \\
\hline 30 & 30 & 90.05 & 63.85 & 89.47 & 143.94 & 79.87 & 51.58 \\
\hline 35 & 40 & 90.05 & 91.67 & 89.46 & 165.37 & 82.47 & 57.46 \\
\hline 40 & 40 & 90.20 & 72.51 & 91.03 & 156.40 & 83.55 & 59.25 \\
\hline
\end{tabular}

(D)

From table I, we can see that the classification accuracy of our method is much better than that of SRC and KSRC when the dimensionality of data is decreased to some lower ones. However, with the growing of dimensionality, the accuracy of our method becomes slightly higher than that of KSRC. But the time consumed of our method is less than that of KSRC obviously. The reason is that we use all the training samples to calculate the kernel function in KSRC. While in our method we only use small number of samples which can replace the whole training samples for each class.

In table $\mathrm{I}(\mathrm{C})$, we can see that when the dimensionality is decreased to 20,the time consumed is less than that in dimensionality of 15 . This is because after selecting 15 "center" samples, some time of dimensionality reduction needs to be consumed while this time is saved in the first condition.

\section{Analysis of experiments.}

In this section, we take the ORL database for example to explain some details of our algorithm.

Firstly, we consider the different methods to construct the "center" samples matrix. The classification accuracy about two methods, including the one described in Algorithm 1 and selecting the samples from the training samples of each class randomly, are listed in table II . Comparing the digits in table II with digits in table I (A), we can see that the classification accuracy of algorithm1 is much higher than that of Gaussian random measurement matrix from the dimensionality of 20 to 80 ,while using the samples selected randomly is not higher than that of the KSRC in most cases. Therefore, the effectiveness of our algorithm to select the "center" samples is confirmed.

TABLE II. CLASSIFICATION ACCURACY ABOUT TWO CONSTRUCTION METHODS OF “CENTER” SAMPLES MATRIX

\begin{tabular}{lccccccc}
\hline & \multicolumn{7}{c}{ Dimionality } \\
\cline { 2 - 8 } methods & 20 & 30 & 40 & 50 & 60 & 70 & 80 \\
\hline Algorithm1 & $\mathbf{9 1 . 6 0}$ & $\mathbf{9 3 . 3 8}$ & $\mathbf{9 3 . 8 2}$ & $\mathbf{9 3 . 0 0}$ & $\mathbf{9 4 . 2 0}$ & $\mathbf{9 4 . 0 0}$ & $\mathbf{9 4 . 3 7}$ \\
random & 85.85 & 89.50 & 91.60 & 92.75 & 92.85 & 93.05 & 94.25 \\
\hline
\end{tabular}

Secondly, in our proposed algorithm, the number of "center" samples is as long as no less than the dimensionality decreased, but with the incensement of "center" samples, more time will be consumed. For example, if the dimensionality required is 20, 40 "center" samples or 80 samples are both feasible. Some experiment results with different number of "center" samples are shown in table III. The classification accuracy with 40 "center" samples is higher than that of 80 "center" samples while the time consumed is less than the latter. We can obtain the conclusion that the number should be selected the lowest one no less than the dimensionality required. If the dimensionality required is 20 , only 40 “center"samples is needed.

TABLE III. EXPERIMENT RESULTS WITH DIFFERENT NUMBER OF “CENTER" SAMPLES

\begin{tabular}{ccccr}
\hline \multirow{2}{*}{ dimensionality } & \multicolumn{2}{c}{40 "center" samples } & \multicolumn{2}{c}{80 “center"samples } \\
\cline { 2 - 5 } & accuracy & time & accuracy & time \\
\hline 20 & $\mathbf{9 1 . 6 0}$ & 9.71 & 91.55 & 9.72 \\
30 & $\mathbf{9 3 . 3 8}$ & 11.32 & 91.83 & 12.30 \\
40 & $\mathbf{9 3 . 8 2}$ & 10.67 & 92.77 & 14.64 \\
\hline
\end{tabular}

Finally, we consider the experiment results when the 
number of training samples in each class is different, as shown in table IV. Two conditions are considered: in each class, there are 5 training samples and 7 training samples respectively. We can know that with the increase of training samples, the classification accuracy will improve and litter time will be consumed.

TABLE IV. EXPERIMENT RESULTS WITH DIFFERENT NUMBER OF TRAINING SAMPLES IN EACH CLASS

\begin{tabular}{cccccc}
\hline & \multirow{2}{*}{$\begin{array}{c}\text { Number } \\
\text { of }\end{array}$} & \multicolumn{2}{c}{ 5samples } & \multicolumn{2}{c}{7 samples } \\
\cline { 3 - 6 } Dim $\begin{array}{c}\text { "center" } \\
\text { samples }\end{array}$ & accuracy & time & accuracy & time \\
\hline 20 & 40 & 91.60 & 9.71 & 94.58 & 5.53 \\
30 & 40 & 93.38 & 11.32 & 95.83 & 6.98 \\
40 & 40 & 93.82 & 10.67 & 96.00 & 7.22 \\
50 & 80 & 93.00 & 13.42 & 96.41 & 8.64 \\
60 & 80 & 94.20 & 13.35 & 96.67 & 12.43 \\
70 & 80 & 94.00 & 18.23 & 95.91 & 15.20 \\
80 & 80 & 94.37 & 17.60 & 95.91 & 12.33 \\
\hline
\end{tabular}

\section{CONCLUSIONS AND FUTURE WORK}

In this paper, a new sparse kernel representation method is proposed for images classification. A small number of training samples were selected to map all training samples and testing sample into feature space and in this space we can classify the testing sample by the basic sparse representation classification algorithm. Some experiments are taken on different databases to test the performance of our proposed method. Comparison about the classification accuracy and the consumed time are made, and the results show that our method can achieve satisfactory classification accuracy without much time consumed. We are planning to further study the sparse kernel presentation in several aspects: 1) adding an algorithm in which the parameter can adjust adaptively; 2) to use this sparse kernel function for clustering and semi-supervised classification; 3) considering adding multi-kernel learning into our algorithm.

\section{ACKNOWLEDGEMENTS}

The authors would like to thank the anonymous reviewers for their constructive comments. This work was supported by the National Science Foundation of China under Grant no. 61072108, 60971112, 60601029, the Basic Science Research Fund in Xidian University under Grant no. JY10000902041 and China Postdoctoral Science Foundation.

\section{REFERENCES}

[1] Saha, S.; Bandyopadhyay, S.; "Unsupervised pixel classification in satellite imagery using a new multiobjective symmetry based clustering approach," TENCON 2008 - 2008 IEEE Region 10 Conference , vol., no., pp.1-6, 19-21 Nov. 2008.

[2] Polat, O.M.; Ozkazanc, Y.S.; , "Material clustering and band reduction in spectral libraries with unsupervised hierarchical classification methods," Signal Processing and Communications Applications (SIU), 2011 IEEE 19th Conference on , vol., no., pp.1081-1084, 20-22 April 2011.

[3] JinHua $\mathrm{Xu}$; Hong Liu; , "Web user clustering analysis based on KMeans algorithm," Information Networking and Automation (ICINA), 2010 International Conference on , vol.2, no., pp.V2-6-V2-9, 18-19 Oct. 2010.
[4] Hongwei Li; Yakui Li; Hanqing Lu; , "Semi-Supervised Learning with Gaussian Processes," Pattern Recognition, 2008. CCPR '08. Chinese Conference on , vol., no., pp.1-5, 22-24 Oct. 2008

[5] Shiming Xiang; Feiping Nie; Changshui Zhang; , "Semi-Supervised Classification via Local Spline Regression," Pattern Analysis and Machine Intelligence, IEEE Transactions on , vol.32, no.11, pp.20392053, Nov. 2010

[6] D Zhang, J.Wang, F.Wang, and C.Zhang: Semi-Supervised Classification with Universum.SDM.pp.323-3 33, 2008.

[7] J. Wright, A. Ganesh, A. Y. Yang, and Y. Ma."Robust face recognition via sparse representation", IEEETransactions on Pattern Analysis and MachineIntelligence, vol.31, no.2, pp: 210-227, Sept.2008.

[8] Demir, B.; Erturk, S.; , "Improving SVM classification accuracy using a hierarchical approach for hyperspectral images," Image Processing (ICIP), 2009 16th IEEE International Conference on , vol., no., pp.28492852, 7-10 Nov. 2009

[9] Cervantes, J.; Xiaoou Li; Wen Yu; , "SVM Classification for Large Data Sets by Considering Models of Classes Distribution," Artificial Intelligence - Special Session, 2007. MICAI 2007. Sixth Mexican International Conference on , vol., no., pp.51-60, 4-10 Nov. 2007.

[10] HUBEL D H,WIESEL T N. Receptive fields of single neurons in the cat's striate cortex[J].Journal of Physiology ,148:574-591,1959.

[11] D L Donoho. Compressed sensing[J].IEEE Trans. on Information theory.52(4):1289-1306, 2006.

[12] M.Aharon,M.Elad,and A.M.Bruckstein,'K-SVD:An Algorithm for designing of overcomplete dictionaries fo sparse representation",IEEE trans. On signal processing,vol,54.no.11,2006.

[13] M.Aharon,M.Elad,'Image denoising via learned dictionaries and sparse representation",CVPR,NY,June 17-22,2006.

[14] Tuomas Virtanen. Separation of sound soueres by convolutive sparse coding. Workshop on Statistical and Perceptional Audio Processing SAPA-2004, Jeju,Korea,Oct.2004.

[15] Pnado Geogriev, Fbaian Theis and Andrzej Cichoeki.Sparse component analysis and blind source separation of inderdetemrined Mixtures. IEEE Transactions on Neural Networks,vol.16,no.4,Jul.2005.

[16] Saeed Gazor and Wei Zhang. Speeeh Enhancement Employing Laplacian-Gaussian Mixture. IEEE Transactions on Speech and Audio Processing, vol.13, no.5, pp.896 - 904, Sep.2005.

[17] Y.Dan, J.J.Atiek, and R.C.Reid. Effieient coding of natural scenes in the lateral Geniculate nueleus:experimental test of a computational theory. Journal of Neuroscience, vol.16, pp.3351-3362, 1996.

[18] J.H.van Hateren and A.van der Schaaf. Independent component filters of natural images compared with simple cells in primary visual cortex. Proceeding of the Royal Society of London, B225,vol.265,pp.359.Mar.1998.

[19] A.Hyvarinen. Gaussian moments for noise in dependent ceomponent analysis. IEEE Signal Proeessing Letters, vol.6, no.6, pp.145$147,1999$.

[20] J. Wright, A. Ganesh, A. Yang, and Y. Ma: Robust face recognition via sparse representation. TPAMI, in press, 2008.

[21] Shenghua Gao, Ivor W. Tsang, Liang-Tien Chia. "Kernel Sparse Representation for Image Classification and Face Recognition,'Proceedings of the 11th European Conference on Computer Vision (ECCV 2010), Crete, Greece, September 2010.

[22] S.Chen,D.Donoho,and M.Saunders, "Atomic decomposition by Basis Pursuit,” SIAM Rev.,vol.43,no.1,pp.129-159,2001.

[23] Thiagarajan, J.J.; Ramamurthy, K.N.; Knee, P.; Spanias, A.; Berisha, V.; , "Sparse representations for automatic target classification in SAR images," Communications, Control and Signal Processing (ISCCSP), 2010 4th International Symposium on, vol., no., pp.1-4, 3-5 March 2010

[24] ZHANG D.SONG F X, XU Y, et al. Advanced patter recognition technologies with applications to biometrics [M]. New York: IGI Global, 2009.

[25] Jain, A.K.; Duin, R.P.W.; Jianchang Mao; , "Statistical pattern recognition: a review," Pattern Analysis and Machine Intelligence, IEEE Transactions on , vol.22, no.1, pp.4-37, Jan 2000.

[26] http://www.face-rec.org/databases/. 
[27] S.S.Chen, D.L.Donoho, M.A.Saunders, Atomic decomposition by basis pursuit, SIAM Review, 2001, 43(1):129-159.

[28] Georghiades, A.S.; Belhumeur, P.N.; Kriegman, D.J.; , "From few to many: illumination cone models for face recognition under variable lighting and pose," Pattern Analysis and Machine Intelligence, IEEE Transactions on, vol.23, no.6, pp.643-660, Jun 2001.
[29] http://www.kernel-machines.org/.

[30] http://yann.lecun.com/exdb/mnist/ 